\title{
Prospective Evaluation of Duodenogastroesophageal Reflux in Gastroesophageal Reflux Disease Patients Refractory to Proton Pump Inhibitor Therapy
}

\section{S. Kunsch A. Neesse T. Linhart C. Nell T.M. Gress V. Ellenrieder}

Department of Gastroenterology, Endocrinology and Metabolism, Faculty of Medicine, Philipps University of Marburg, Marburg, Germany

Key Words

GERD $\cdot$ DGERD $\cdot$ Proton pump inhibitor $\cdot$ Bilitec with a limited effect of PPIs on reducing DGER. Thus, persistent DGER may play a key role in mediating reflux symptoms refractory to high-dose PPIs. Copyright $\Theta 2012$ S. Karger AG, Basel

\section{Introduction}

Gastroesophageal reflux disease (GERD) is defined as the presence of symptoms or lesions that can be attributed to the reflux of gastric contents into the esophagus. It represents one of the most common disorders in western countries affecting up to $35 \%$ of the adult population [1]. Heartburn and acid regurgitation are the typical clinical symptoms in GERD patients and reflux esophagitis represents the most common morphological correlate [2]. GERD has been shown to have a significant impact on health-care utilization and patient's quality of life $[3,4]$. The introduction of proton pump inhibitors (PPIs) has entirely revolutionized the medical management of GERD [5-7]. PPIs are well tolerated and superior to any other established medical treatment strategy including $\mathrm{H}_{2}$-receptor antagonists in achieving both symptom control and mucosal healing rates [8]. In fact, complete healing of erosive lesions and symptoms control

Dr. Volker Ellenrieder

Department of Gastroenterology, Endocrinology and Metabolism

Faculty of Medicine, Philipps University of Marburg, Baldingerstrasse

DE-35041 Marburg (Germany)

E-Mail ellenrie@med.uni-marburg.de 
have been reported in up to $90 \%$ of the patients [9]. However, despite the general efficacy of PPI treatment in reflux patients there is now cumulating evidence indicating a subgroup of patients with incomplete therapy responses and persistent reflux symptoms, even under a high-dose regimen. Currently, between 10 and $40 \%$ of GERD patients might remain symptomatic despite adequate PPI therapy [10-12], and this treatment failure can only partially be attributed to insufficient enzymatic metabolism of the drug. This subgroup of patients represents an unresolved clinical challenge since no standardized and validated therapy alternatives are currently available.

In particular, the impact of DGER in symptomatic GERD patients under PPI therapy remains a matter of debate. DGER is defined as reflux of duodenal contents into the stomach and esophagus containing duodenal secretes, pancreatic enzymes and bile acid. Currently, there is neither a validated nor an approved therapeutic regimen for the treatment of DGER, and the precise underlying mechanisms of action of PPIs in reducing DGER are still not established. It has been suggested that the effects of PPIs on DGER are only indirect, mediated by the ability of PPIs to decrease not only gastric acidity but also the volume of gastric juice [13]. Interestingly, a good correlation between total bilirubin content and the concentrations of pancreatic enzymes in the refluxate has been reported, suggesting that bilirubin is a good marker for DGER. To this end, a fiberoptic spectrophotometric diagnostic device (Bilitec ${ }^{\circledR} 2000$ ) has been developed to quantify bile reflux in an ambulatory setting similar to the 24-hour $\mathrm{pH}$ measurement [14]. The available studies investigating DGER in PPI nonresponders yielded conflicting results. For instance, Tack et al. [15] identified DGER as an important underlying course in patients that poorly responded to PPIs either on a single or double dose. Using the GABA(B) agonist Baclofen, the same group reported a reduction of DGER and reflux-related symptoms, further underscoring a causative impact of DGER on clinical symptoms refractory to PPI treatment [16]. By contrast, a recent comparison of clinical nonresponders and responders on a single-dose PPI therapy revealed no differences with respect to the amount of DGER [17]. Notably, all available studies are limited by the fact that reflux measurements prior to the initiation of PPI treatment were not performed. This raises the question whether high-dose PPI treatment is capable of reducing DGER significantly in patients without entire symptom control under therapy. Therefore, the aim of this study was to compare the degree of DGER and esophageal acid exposure in GERD patients who continued to be symptomatic (PPI nonresponders) with those who were successfully treated with PPI twice daily (PPI responders) to glean insights into the pathophysiology mediating PPI failure. To address this question for the first time, we performed $\mathrm{pH}$ and Bilitec measurement as well as upper endoscopy before and under therapy with high-dose PPIs.

\section{Materials and Methods}

Patients and Study Protocol

85 patients were recruited to the study. All patients were referred to the outpatients department of the University of Marburg due to suspected reflux disease. All patients had a history of PPI treatment on single or double dose for at least 4 weeks within the last 12 months without entire symptom control. The pattern of clinical symptoms was evaluated with a questionnaire, specifically designed in our group. The specification of the symptoms heartburn, regurgitation, chest pain, dysphagia, nocturnal coughing, hoarseness and vomiting were graded into 1 : not existing, 2 : mild, 3: moderate, and 4: strong [18]. Only patients with the typical clinical signs of reflux disease such as heartburn and regurgitation with a frequency of at least 3 episodes of symptoms per week were enrolled in the study. Patients meeting inclusion criteria were asked to provide a written informed consent before enrolment. Exclusion criteria were previous surgery of the upper GI tract, the history of Barrett's esophagus, intake of GI motility affecting medication or history of Helicobacter pylori infection. Furthermore, patients unable to complete the protocol or with a positive $H$. pylori test were excluded from the study.

All patients included underwent combined 24-hour $\mathrm{pH}$ measurement and Bilitec 2000 procedure, esophageal manometry to exclude oesophageal motility disorders, upper GI endoscopy and $H$. pylori testing. If existing, an acid suppression therapy was withdrawn at least 7 days prior TO diagnostic evaluation. Only patients with an elevated reflux in $\mathrm{pH}$ and/or Bilitec measurement were further studied. Pantoprazole $40 \mathrm{mg}$ twice daily (orally) was applied for 8 weeks. Under therapy, clinical symptoms were reevaluated and the 24-hour $\mathrm{pH}$ and Bilitec 2000 measurement as well as upper endoscopy were repeated. Under treatment patients were grouped in PPI non-responders when having at least 3 episodes of symptoms like heartburn or regurgitation under pantoprazole $80 \mathrm{mg}$ per day. The diagram of the study design is displayed in figure 1 . The study protocol was approved by the local ethics committee.

Upper GI Endoscopy

All patients were subjected to upper GI endoscopy. In addition, the presence of esophagitis was determined and graded according to the classification of Savary and Miller. The presence of hiatal hernia was measured endoscopically and classified into large $(>3$ $\mathrm{cm})$ and small $(<3 \mathrm{~cm})$. The distal portion of the esophagus was carefully evaluated to determine the presence of Barrett's esophagus. In patients with suspected Barrett's epithelium, four quadrant biopsy specimens were taken for histological examination. H. pylori infection was determined by biopsy urease test in all cases. 


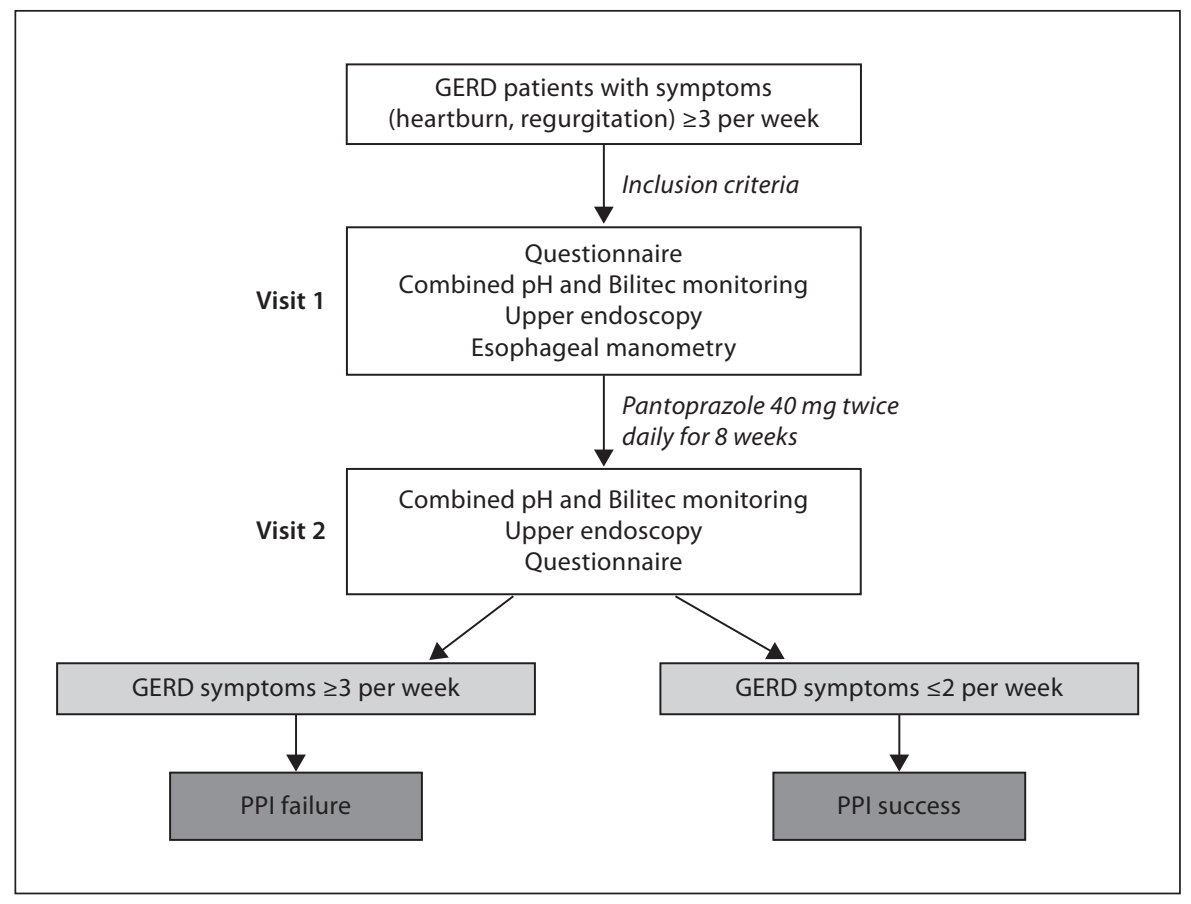

Fig. 1. Diagram of study design.

pH Monitoring and Measurement of DGER by Bilitec 2000

Ambulatory esophageal $\mathrm{pH}$ monitoring was performed using an antimony $\mathrm{pH}$ electrode with a separate skin reference electrode (Medtronic, Germany). Data were stored on a portable digital recorder (Digitrapper Mk III). Prior to analysis, the $\mathrm{pH}$ probe was calibrated in buffer solutions of $\mathrm{pH} 7$ and 1 . The catheter was introduced nasally and positioned $5 \mathrm{~cm}$ above the lower esophageal sphincter. An episode of acid reflux was defined as a decrease in esophageal $\mathrm{pH}$ value below 4 for at least $10 \mathrm{~s}$. A pathological $\mathrm{pH}$ measurement was defined for a time with $\mathrm{pH}$ values below 4 for longer than $4 \%$ of the total 24 -hour period $[15,19]$.

The fiber-optic spectrophotometer Bilitec 2000 was used to quantify DGER. The system consists of a miniaturized probe of $1.5 \mathrm{~mm}$ in diameter that beams light signals into the esophagus and back via a plastic fiberoptic bundle. Prior to analysis, the probe was calibrated in water. The catheter was introduced nasally and positioned $5 \mathrm{~cm}$ above the lower esophageal sphincter. A symptom episode of DGER was defined as an increase in esophageal bilirubin OD to 0.14 for at least $10 \mathrm{~s}$. Pathological values were defined for a time with an OD above 0.14 for longer than $4.6 \%$ of the total 24-hour period. A specialized diet was prescribed to exclude an interference with DGER measurement $[15,19]$.

\section{Esophageal Manometry}

Each patient underwent standard esophageal manometry using an 8-channel solid-state catheter (Medtronic Inc., Germany). The catheter was introduced nasally. Patients were studied in the supine position after an overnight fast. Swallows were detected by direct visualization. The sensors, spaced $5 \mathrm{~cm}$ apart, were used to record resting lower esophageal sphincter pressure and distal esophageal wave contraction amplitude. Resting intragastric pressure was used as a zero reference for all data acquired. Resting lower esophageal sphincter pressure (LESP) was measured at end expiration. Recording sites for evaluation of esophageal peristalsis were located $3,8,13$ and $18 \mathrm{~cm}$ above the upper point of the lower esophageal sphincter. Esophageal peristalsis was assessed with 10 wet swallows of $5 \mathrm{ml}$ of water at room temperature.

\section{Symptom Association}

Patients were instructed to document the presence of the GERD-related symptoms heartburn and acid regurgitation by using an event marker and recording in a diary. Association of documented symptoms with acidic reflux or DGER was estimated if symptoms occurred within a 5-min interval of a reflux event (prior to or following a reflux event).

\section{Statistics}

Data are reported as mean \pm SD or median. Exposure time of the distal esophagus to bilirubin was compared using the MannWhitney $\mathrm{U}$ test. $\mathrm{p}<0.05$ was considered significant.

\section{Results}

\section{Clinical Observation}

Overall, 85 patients with typical reflux symptoms such as heartburn and/or acid regurgitation and a history of ineffective PPI treatment were screened. Twenty-three patients were excluded from the study due to early withdrawal $(n=10)$, a positive $H$. pylori test $(n=7)$ or presence of Barrett's esophagus $(n=6)$. Additional 15 patients were 
Fig. 2. Flow chart of the results of the

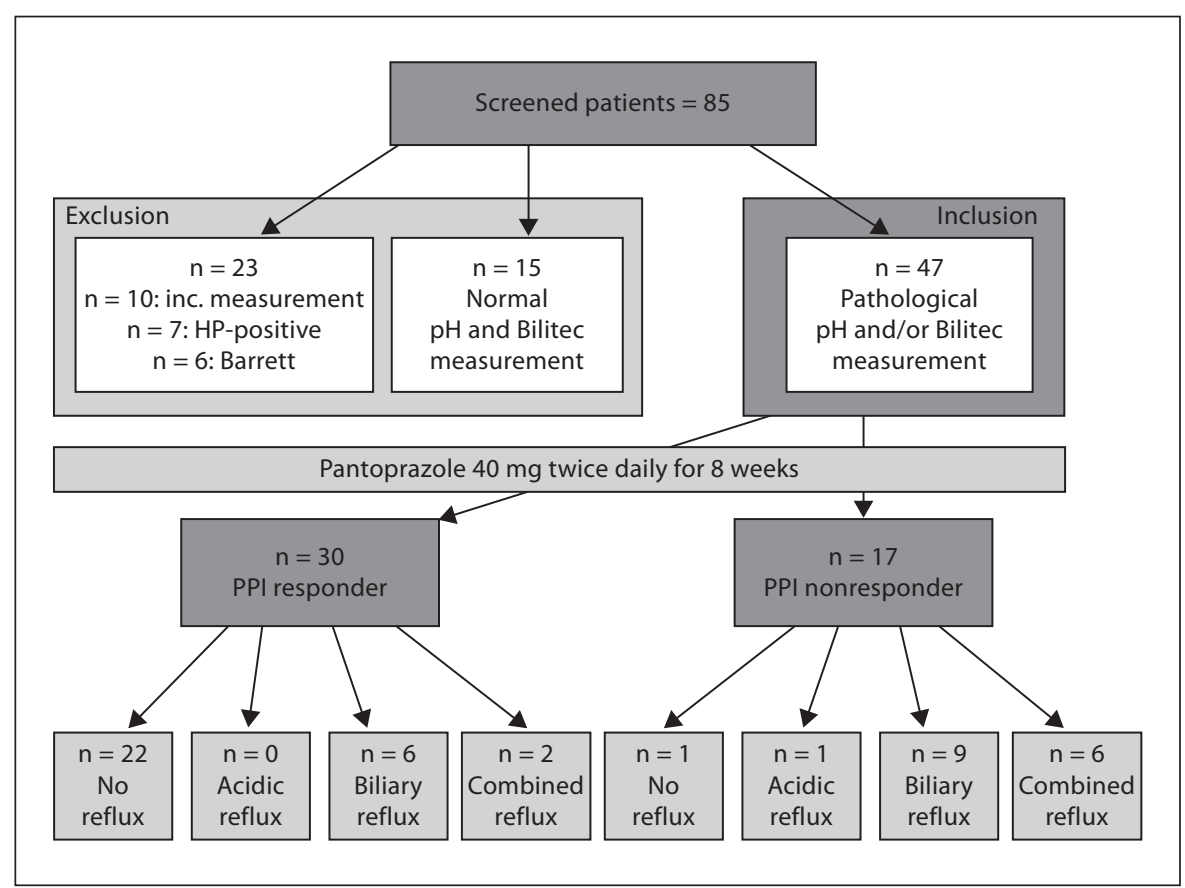
study.

excluded owing to normal $\mathrm{pH}$ and Bilitec measurements. Forty-seven patients completed the study and were treated with $40 \mathrm{mg}$ pantoprazole twice daily according to clinical responsiveness under therapy. Patients were divided into PPI responder ( $\leq 2$ episodes per week) and PPI nonresponder ( $\geq 3$ episodes per week). For further analysis, PPI responder $(n=30)$ and nonresponder $(n=17)$ were analyzed separately. The results of the study are displayed in figure 2. The demographic and clinical data of patients are presented in table 1 . All patients $(n=47)$ had a history of previous PPI treatment. The majority of patients were male in both groups. There were no significant differences with respect to duration of symptoms, BMI, history of cholecystectomy and alcohol and cigarette consumption. Age was the only statistically significant parameter between PPI responders and nonresponders. The PPI success group was significantly older than the PPI failure group ( $52 \pm 12$ vs. $43 \pm 11$ years, $\mathrm{p}<0.05)$, respectively.

\section{DGER and Acidic Reflux}

No significant differences between the PPI failure and success groups concerning the degree of acidic (11.5 \pm 10.7 vs. $7.1 \pm 9.1 \%$; p > 0.05) and biliary reflux (24.5 \pm 18.6 vs. $22.8 \pm 22.8 \%$; p $>0.05$ ) were detected prior to treatment with pantoprazole $80 \mathrm{mg}$ daily. Under treatment, abnormal $\mathrm{pH}$ test was present in $2 / 30(7 \%)$ of the
Table 1. Demographic and clinical characteristics of study patients

\begin{tabular}{lcc}
\hline & $\begin{array}{l}\text { PPI failure } \\
\text { group }\end{array}$ & $\begin{array}{l}\text { PPI success } \\
\text { group }\end{array}$ \\
\hline $\begin{array}{l}\text { Number } \\
\text { Mean duration of symptoms } \pm \text { SD, }\end{array}$ & 17 & 30 \\
$\quad$ years & $7.2 \pm 3.8$ & $6.1 \pm 27$ \\
History of PPI therapy, \% & 100 & 100 \\
Gender, M/F & $11 / 9$ & $18 / 15$ \\
Mean age \pm SD, years & $43 \pm 11^{*}$ & $52 \pm 12^{*}$ \\
Mean BMI \pm SD (range) & $27.9 \pm 5$ & $26.9 \pm 52$ \\
& $(22-38)$ & $(20-37)$ \\
Regular alcohol user, \% & 35 & 33 \\
Smoker, \% & 29 & 30 \\
History of cholecystectomy, \% & 12 & 7 \\
\hline
\end{tabular}

* Significant differences with $\mathrm{p}<0.05$.

PPI success group and 7/17 (41\%) of the PPI failure group. Elevated DGER was detected in $8 / 30(27 \%)$ patients of the PPI success group and in 15/17 (88\%) of the PPI failure group.

Interestingly, treatment with pantoprazole significantly reduced both acidic ( $7.1 \pm 9.1$ vs. $2.4 \% \pm 3.8 ; \mathrm{p}<$ $0.05)$ and biliary reflux (22.8 \pm 22.8 vs. $6.6 \pm 10.8 \%$; $p<$ 


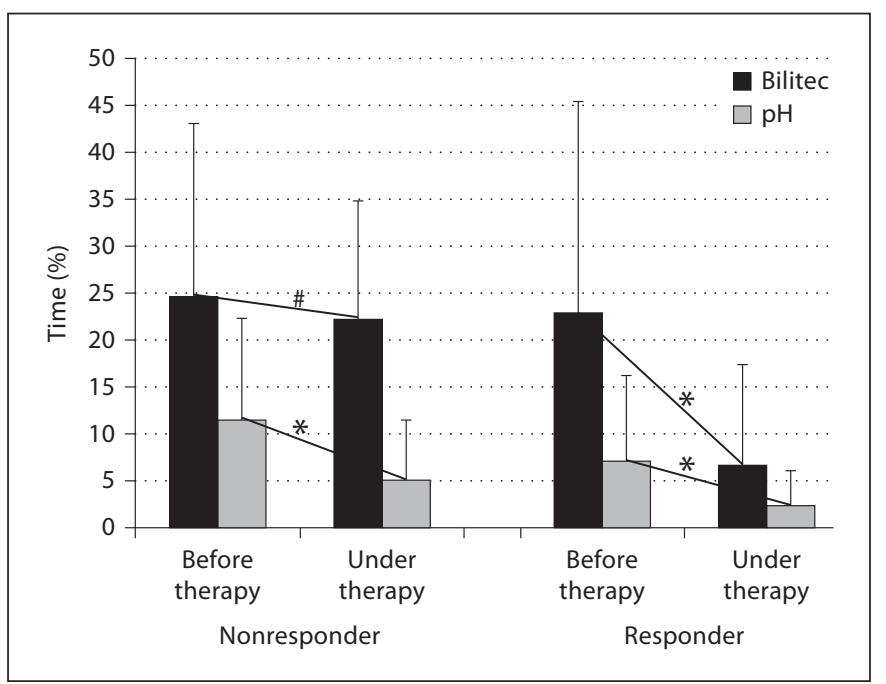

Fig. 3. Comparison of acidic reflux (pH) and DGER (Bilitec) of PPI responders and nonresponders before and under therapy with pantoprazole. Values are displayed in fraction time (\%) of 24-hour measurements. ${ }^{*} \mathrm{p}<0.05 ;{ }^{\#} \mathrm{p}>0.05$.

0.05) in PPI success group patients. Furthermore, only acidic reflux could be reduced significantly in patients of the PPI failure group $(11.5 \pm 10.7$ vs. $5.1 \pm 6.4 \%$; $p<0.05)$. In contrast, DGER was not significantly reduced in this group (24.5 \pm 18.6 vs. $22.2 \pm 12.7 \%$; p > 0.05) (fig. 3$)$. In this line of evidence, number of acidic and biliary reflux events were significantly reduced in the PPI success group $(117.2 \pm 65.5$ vs. $51.5 \pm 57$ acidic reflux events and 52.2 \pm 54.1 vs. $26.2 \pm 33.8 \%$ biliary reflux events). In the PPI failure group, only the number of acidic reflux events was significantly diminished $(176.5 \pm 125.2$ vs. $105.2 \pm$ 98.8). However, the number of biliary reflux events was not reduced significantly in this group $(75.5 \pm 51.3$ vs. $62.6 \pm 36.1 ; \mathrm{p}>0.05)$. These findings are illustrated in figure 4 . Consequently, the reduced clinical benefit of treatment with pantoprazole was accompanied by a limited reduction of DGER under therapy compared to the level before therapy.

\section{Endoscopic Findings}

All patients underwent upper endoscopy before and under treatment with pantoprazole. If existing, reflux esophagitis was classified according to the classification of Savary and Miller.

Before treatment, 10 (33\%) responders had negative endoscopy and 20 (67\%) showed signs of erosive esophagitis (grade 1: 11, grade 2: 8, grade 3: 1). Seven (41\%) non-

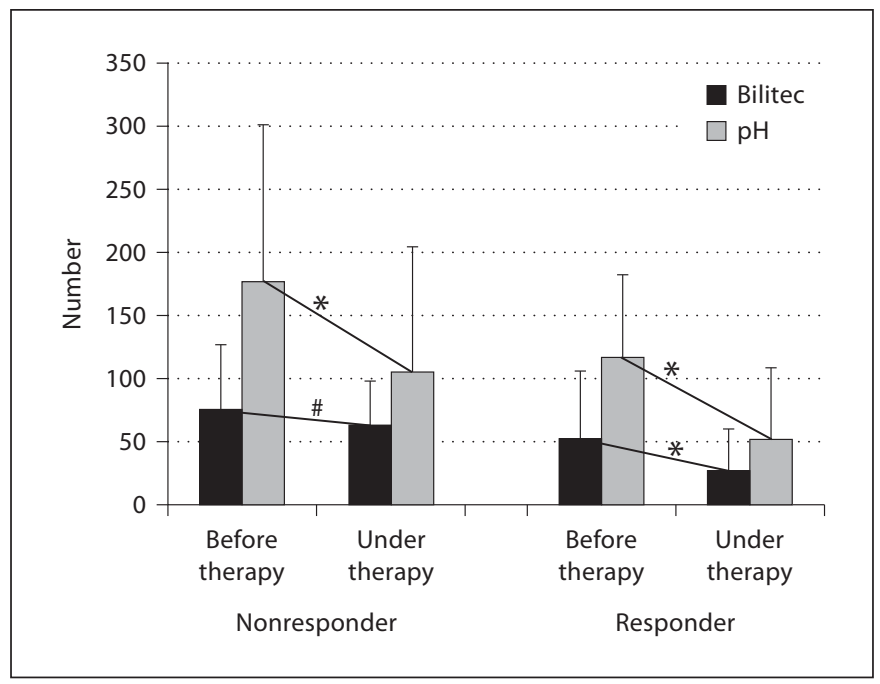

Fig. 4. Comparison of acidic ( $\mathrm{pH})$ and biliary (Bilitec) reflux events of PPI responders and nonresponders before and under therapy with pantoprazole. Values are displayed in average number of 24 -hour measurements. ${ }^{*} \mathrm{p}<0.05 ;{ }^{*} \mathrm{p}>0.05$.

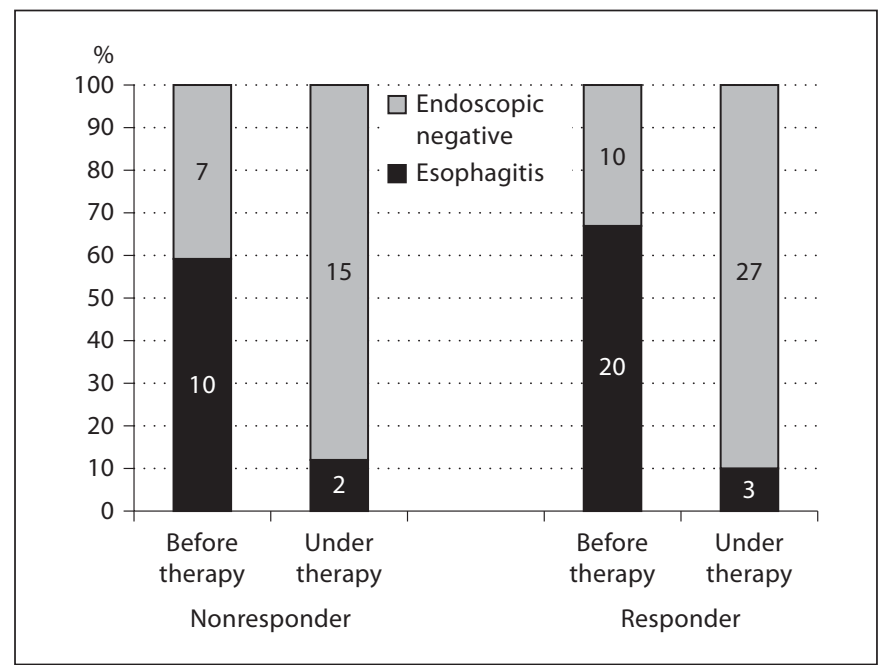

Fig. 5. Comparison of endoscopic findings of PPI responders and nonresponders before and under therapy with pantoprazole. No significant differences could be detected between responders and nonresponders.

responders had negative endoscopy and 10 (59\%) erosive esophagitis (grade 1: 4 , grade 2: 4 , grade 3:2). No significant differences between both groups could be detected in the statistical analysis. The majority of patients in both groups had normal endoscopy under therapy (PPI failure: 
15/17 (88\%); PPI success: 27/30 (90\%)). Erosive esophagitis could be detected in 2 of 17 (12\%) patients with PPI failure and in 3 of 30 (10\%) responders under therapy, respectively. According to the classification by Savary and Miller, only grade 1 esophagitis was detected in both groups. In the statistical analysis, no differences were found between both groups (fig. 5). The presence of hiatal hernia was quantified endoscopically. In the PPI nonresponder group 13 of 17 (76.5\%) patients had hiatal hernia (6 large, 7 small), whereas 20 of 30 (67\%) PPI responders had signs of hiatal hernia (7 large, 13 small).

\section{Manometry Findings}

Esophageal manometry was performed to localize the lower esophageal sphincter and rule out severe motility disorders. Regarding the pressure of the lower esophageal sphincter, no significant differences between the PPI failure and success groups were noted (10.8 \pm 5 vs. $11.8 \pm$ $4 \mathrm{~mm} \mathrm{Hg}$ ). Patients in the PPI failure group appeared to have lower contraction amplitude in the distal esophagus $(53.9 \pm 25.4$ vs. $64 \pm 32.8 \mathrm{~mm} \mathrm{Hg})$, but these differences did not reach significance in the statistical analysis.

\section{Symptom Analysis}

Under treatment, 42 episodes of either heartburn or acid regurgitation were recorded during the measurement of $\mathrm{pH}$ and Bilitec in the PPI failure group. In the PPI success group no symptomatic episodes were recorded under therapy. A temporal correlation with reflux events was performed, showing that only 12 (28\%) episodes occurred independent from reflux events, whereas 26 events (62\%) were associated with either isolated DGER $(\mathrm{n}=14$, $33 \%)$ or with combined reflux events (DGER and acidic reflux) $(\mathrm{n}=12,29 \%)$. Only $4(10 \%)$ symptomatic events were related to isolated acidic reflux events.

\section{Discussion}

Clinical trials have shown significant higher levels of DGER in patients with a Barrett's mucosa compared to uncomplicated GERD [20]. However, the available data on the clinical response of GERD patients regarding DGER are ambiguous and seem to depend on different inclusion criteria and treatment schedules (e.g. singledose vs. high-dose pantoprazole) $[15,21]$. A previous study from our group has shown that only in about $50 \%$ of patients with combined pathological reflux (bile and acid) DGER could be normalized by high-dose pantoprazole [19]. Here, we compare the degree of DGER and esophageal acid exposure in a cohort of GERD patients who had a history of incomplete symptom control with PPIs during the past year.

Under treatment with high-dose pantoprazole for 8 weeks, the majority (88\%) of PPI nonresponders revealed pathological levels of DGER compared to only $27 \%$ of clinical responders. This result is further supported by a significantly higher degree of DGER in the PPI failure group compared to the PPI success group. However, in line with Gasiorowska et al. [17], we also detected elevated levels of DGER in a subgroup of PPI responders. This raises the question whether DGER is merely an epiphenomenon of PPI treatment rather than a causative mechanism of PPI nonresponse. To answer this crucial question, we measured acidic and biliary reflux prior to PPI therapy. Interestingly, no statistically significant difference could be found between PPI responders and nonresponders regarding acidic and biliary reflux before treatment. This finding clearly rules out a general effect of PPI therapy on DGER. Interestingly, high-dose PPI treatment significantly reduced acidic reflux in both groups and biliary reflux only in the PPI responders. Thus, the presented study firstly describes the association of PPI nonresponse with the inability of acid suppression therapy to reduce DGER. The precise mechanisms mediating persistent DGER under PPI treatment are an unsolved issue up to date. As we ruled out esophageal motility disorders, disturbance of gastroduodenal motility could lead to increased reflux of bile and other components of duodenal juice into the esophagus. Further analyses aiming on measurement of antroduodenal motility are required to answer this important issue.

Strikingly, the endoscopy results of our study showed that $12 \%$ in the PPI failure group and $10 \%$ in the PPI success group had reflux esophagitis under therapy. Significant differences between both groups could not be detected before and after therapy. Therefore, we denote a significant impact on the healing rate of reflux esophagitis independently of (1) a persistent biliary reflux and (2) symptom relief under therapy. Traditionally, mucosal inflammation has been regarded as a hallmark feature in the development of Barrett's esophagus. On a cellular level, destruction of the mucosal barrier and increasing permeability of the cell membrane for hydrogen ions occur after exposure to bile salts, and molecular analysis has revealed that bile acids induce overexpression of mitogen factors like cox-2 and c-myc in the esophageal mucosa [22-24].

The fact that patients with Barrett's esophagus show higher levels of DGER [20], taken with our observation that endoscopic lesions do not occur more frequently in 
the PPI nonresponder group with significantly higher levels of DGER, casts doubt on this progression model and may suggest alternative routes to Barrett's esophagus depending on the predominant type of reflux. The concept of esophageal mucosa dilated intercellular spaces (DIS) has been recently introduced and may play a role in impairing mucosal integrity following exposure to acid and weakly acidic solutions even without endoscopically visible signs of inflammation [25].

Our study has several shortcomings that should be discussed. First, the number of patients included was relatively small as we only enrolled patients with a history of ineffective PPI therapy in the past year. Therefore, our study only investigates about $10-40 \%$ of the most problematic and clinically challenging GERD patients. Consequently, the frequency of PPI nonresponders with elevated DGER has to be considered much lower in a general population of GERD patients. Furthermore, the impact of acidic reflux in generating GERD-related symptoms could not be clearly identified in this study. Although acidic reflux was not significantly different in responders and nonresponders, there was still a tendency towards increased acidic reflux in the nonresponder cohort. Furthermore, a potential limitation of our study is the fact that we applied the Bilitec device to investigate DGER. The combined multichannel intraluminal im- pedance and $\mathrm{pH}$ measurement (MII-pH) represents a novel technology to detect and quantify nonacid or weakly acidic reflux events $[26,27]$. The direction and proximal extent of refluxed liquid or gas can be measured regardless of its hydrogen ion content, and the diagnostic impact is superior to the conventional $\mathrm{pH}$ measurement [28]. In studies using MII-pH, a clear shift towards more weakly or nonacidic reflux was noted in GERD patients who failed to respond to PPI twice daily $[29,30]$. Nevertheless, it could be demonstrated that nonacid reflux and DGER detected by the Bilitec device are two separate phenomena [31], and therefore, MII-pH measurement cannot replace Bilitec 2000.

To conclude, our study shows that the degree of DGER is significantly higher in GERD patients refractory to high-dose PPI treatment compared to those with symptom relief under therapy. Furthermore, PPI nonresponse is associated with the inability of acid suppression therapy to reduce DGER significantly. Thus, persistent DGER may play a key role in mediating reflux symptoms refractory to high-dose PPIs.

\section{Acknowledgement}

This study was supported by a Research Grant of the University Medical Center Giessen and Marburg (UKGM) Germany.

\section{References}

1 Tytgat GN, McColl K, Tack J, Holtmann G, Hunt RH, Malfertheiner P, Hungin AP, Batchelor HK: New algorithm for the treatment of gastro-oesophageal reflux disease. Aliment Pharmacol Ther 2008;27:249-256.

-2 Shaker R, Castell DO, Schoenfeld PS, Spechler SJ: Nighttime heartburn is an under-appreciated clinical problem that impacts sleep and daytime function: the results of a Gallup survey conducted on behalf of the American Gastroenterological Association. Am J Gastroenterol 2003;98:1487-1493.

$\checkmark 3$ Charbel S, Khandwala F, Vaezi MF: The role of esophageal $\mathrm{pH}$ monitoring in symptomatic patients on PPI therapy. Am J Gastroenterol 2005; 100:283-289.

4 Dent J, El-Serag HB, Wallander MA, Johansson S: Epidemiology of gastro-oesophageal reflux disease: a systematic review. Gut 2005; 54:710-717.

5 Dekel R, Morse C, Fass R: The role of proton pump inhibitors in gastro-oesophageal reflux disease. Drugs 2004;64:277-295.

6 Coron E, Hatlebakk JG, Galmiche JP: Medical therapy of gastroesophageal reflux disease. Curr Opin Gastroenterol 2007;23:434439.
7 Richter JE: The many manifestations of gastroesophageal reflux disease: presentation, evaluation, and treatment. Gastroenterol Clin North Am 2007;36:577-5ix.

8 van PB, Numans ME, Bonis PA, Lau J: Shortterm treatment with proton pump inhibitors, $\mathrm{H}_{2}$-receptor antagonists and prokinetics for gastro-oesophageal reflux diseaselike symptoms and endoscopy negative reflux disease. Cochrane Database Syst Rev 2004;CD002095.

-9 Moayyedi P, Talley NJ: Gastro-oesophageal reflux disease. Lancet 2006;367:2086-2100.

10 Fass R, Mackel C, Sampliner RE: 24-hour pH monitoring in symptomatic patients without erosive esophagitis who did not respond to antireflux treatment. J Clin Gastroenterol 1994;19:97-99.

11 Fass R, Shapiro M, Dekel R, Sewell J: Systematic review: proton-pump inhibitor failure in gastro-oesophageal reflux disease - where next? Aliment Pharmacol Ther 2005;22:7994
12 Klinkenberg-Knol EC, Nelis F, Dent J, Snel P, Mitchell B, Prichard P, Lloyd D, Havu N, Frame MH, Roman J, Walan A: Long-term omeprazole treatment in resistant gastroesophageal reflux disease: efficacy, safety, and influence on gastric mucosa. Gastroenterology 2000;118:661-669.

13 Tack J: The role of bile and pepsin in the pathophysiology and treatment of gastro-oesophageal reflux disease. Aliment Pharmacol Ther 2006;24(suppl 2):10-16.

14 Vaezi MF, Lacamera RG, Richter JE: Validation studies of Bilitec 2000: an ambulatory duodenogastric reflux monitoring system. Am J Physiol 1994;267:G1050-G1057.

15 Tack J, Koek G, Demedts I, Sifrim D, Janssens J: Gastroesophageal reflux disease poorly responsive to single-dose proton pump inhibitors in patients without Barrett's esophagus: acid reflux, bile reflux, or both? Am J Gastroenterol 2004;99:981-988.

16 Koek GH, Sifrim D, Lerut T, Janssens J, Tack J: Effect of the GABA(B) agonist baclofen in patients with symptoms and duodeno-gastro-oesophageal reflux refractory to proton pump inhibitors. Gut 2003;52: 1397-1402. 
17 Gasiorowska A, Navarro-Rodriguez T, Wendel C, Krupinski E, Perry ZH, Koenig K, Moty B, Powers J, Fass R: Comparison of the degree of duodenogastroesophageal reflux and acid reflux between patients who failed to respond and those who were successfully treated with a proton pump inhibitor once daily. Am J Gastroenterol 2009;104:20052013.

-18 Kunsch S, Linhart T, Fensterer H, Adler G, Gress TM, Ellenrieder V: Prevalence of a pathological DGER (duodeno-gastric-oesophageal reflux) in patients with clinical symptoms of reflux disease. Z Gastroenterol 2008;46:409-414.

-19 Kunsch S, Neesse A, Linhart T, Steinkamp M, Fensterer H, Adler G, Gress TM, Ellenrieder V: Impact of pantoprazole on duodenogastro-esophageal reflux (DGER). Z Gastroenterol 2009;47:277-282.

20 Vaezi MF, Richter JE: Synergism of acid and duodenogastroesophageal reflux in complicated Barrett's esophagus. Surgery 1995;117: 699-704.

-21 Koek GH, Sifrim D, Lerut T, Janssens J, Tack $\mathrm{J}$ : Effect of the GABA(B) agonist baclofen in patients with symptoms and duodeno-gastro-oesophageal reflux refractory to proton pump inhibitors. Gut 2003;52:1397-1402.
22 Schweitzer EJ, Bass BL, Batzri S, Harmon JW: Bile acid accumulation by rabbit esophageal mucosa. Dig Dis Sci 1986;31:1105-1113.

23 Tselepis C, Morris CD, Wakelin D, Hardy R, Perry I, Luong QT, Harper E, Harrison R, Attwood SE, Jankowski JA: Upregulation of the oncogene c-myc in Barrett's adenocarcinoma: induction of c-myc by acidified bile acid in vitro. Gut 2003;52:174-180.

24 Zhang F, Altorki NK, Wu YC, Soslow RA, Subbaramaiah K, Dannenberg AJ: Duodenal reflux induces cyclooxygenase- 2 in the esophageal mucosa of rats: evidence for involvement of bile acids. Gastroenterology 2001;121:1391-1399.

25 Farre R, Fornari F, Blondeau K, Vieth M, De VR, Bisschops R, Mertens V, Pauwels A, Tack J, Sifrim D: Acid and weakly acidic solutions impair mucosal integrity of distal exposed and proximal non-exposed human oesophagus. Gut 2010;59:164-169.

26 Castell DO, Vela M: Combined multichannel intraluminal impedance and pH-metry: an evolving technique to measure type and proximal extent of gastroesophageal reflux. Am J Med 2001;111(suppl 8A):157S-159S.
27 Weigt J, Monkemuller K, Kolfenbach S, Malfertheiner P: Standards and innovations in the diagnosis of gastroesophageal reflux disease. Z Gastroenterol 2007;45:1141-1149.

28 Wise JL, Murray JA: Utilising multichannel intraluminal impedance for diagnosing GERD: a review. Dis Esophagus 2007;20:8388.

29 Zerbib F, Roman S, Ropert A, des Varannes SB, Pouderoux P, Chaput U, Mion F, Verin E, Galmiche JP, Sifrim D: Esophageal pH-impedance monitoring and symptom analysis in GERD: a study in patients off and on therapy. Am J Gastroenterol 2006;101:19561963.

30 Vela MF, Camacho-Lobato L, Srinivasan R, Tutuian R, Katz PO, Castell DO: Simultaneous intraesophageal impedance and $\mathrm{pH}$ measurement of acid and nonacid gastroesophageal reflux: effect of omeprazole. Gastroenterology 2001;120:1599-1606.

-31 Pace F, Sangaletti O, Pallotta S, Molteni P, Porro GB: Biliary reflux and non-acid reflux are two distinct phenomena: a comparison between 24-hour multichannel intraesophageal impedance and bilirubin monitoring. Scand J Gastroenterol 2007;42:1031-1039. 